\title{
Retinal Fold
}

National Cancer Institute

\section{Source}

National Cancer Institute. Retinal Fold. NCI Thesaurus. Code C124578.

A crease in the retina. 